\title{
Interruptions and multi-tasking: moving the research agenda in new directions
}

\section{Johanna I Westbrook}

\section{Correspondence to}

Professor Johanna I Westbrook, Centre for Health Systems and Safety Research, Australian Institute of Health Innovation, University of New South Wales Medicine, Kensington, NSW 2052, Australia;

J.Westbrook@unsw.edu.au

Accepted 8 July 2014 Published Online First 18 July 2014

\section{SLinked}

http://dx.doi.org/10.1136/ bmjqs-2013-002484

\section{CrossMark}

To cite: Westbrook JI. BMJ Qual Saf 2014;23:877-879.
When the media-savvy US psychiatrist Edward Hallowell first asked people to imagine how they would perform on a tennis court with two, three or even more balls in play, it seemed obvious that, no matter how talented or fit, everyone would be hard pressed to keep up, let alone execute any precision shots. ${ }^{1}$ Dr Hallowell's book, CrazyBusy, went a long way towards raising popular awareness of something that many people felt intuitively. That is, that multi-taskingand the associated interruptions that demand we either switch between competing tasks or load one task on top of another, say by talking on the phone while responding to emails-really was contributing to feeling frantic.

Yet, since the book's publication in 2006, we've just kept raising the 'busyness' bar in our daily personal and professional lives. Email ushered in expectations that the instantaneous delivery of messages would elicit similarly instant responses. Now, in the communications-saturated environment of texting, Instagram, Snapchat, Tumblr, Facebook and Twitter, frequent interruptions and multi-tasking are so familiar that the absence of instant 'connectivity' can cause mild anxiety-or, for some of us, dismay and distress. But, despite the many unquestionable benefits of extraordinary connectivity, and the unprecedented access to information and interaction it enables, the cognitive and social demands of the rapidly evolving digital communication landscape come at a cost. In healthcare organisations, the relentless, competing demands for clinicians' attention are not merely another source of workplace stress, but represent an issue that goes to the very heart of patient safety and quality of care.

Studying communication patterns in the healthcare sector is not only vitalit's fascinating. Healthcare delivery is largely reliant on the flow of clear, concise and accurate information across the many complex webs that link patients, different healthcare professionals and support staff via paper- and computer-based systems. Breakdowns in communication have consistently been found to be associated with negative outcomes and errors. ${ }^{2}{ }^{3}$ Yet the very human character of the clinical frontline means interruptions and multi-tasking are inevitable. Clinicians in emergency departments experience 6-7 interruptions per hour, and general ward staff around $3-4 .{ }^{4} 5$ Direct observational studies show that doctors, often in response to interruptions, spend around $20 \%{ }^{6}$ of their time multi-tasking and nurses $6 \%{ }^{7}$ Most frequently this involves talking or listening while also attending to a related or unrelated task such as documenting or performing direct care.

Staff complain about the frequency of interruptions and identify them as contributing factors in workplace errors. ${ }^{89}$ In one study nurses in two hospitals were shown to make significantly more errors, and more serious errors, as interruptions increased. ${ }^{10}$ This is consistent with the findings of many psychological studies in the laboratory that demonstrate a range of negative outcomes associated with interruptions. These include more errors, forgetting to complete tasks, taking longer to complete tasks ${ }^{11-13}$ and experiencing stress and anxiety. ${ }^{14}$ However, the relationship between interruptions and errors is by no means straightforward. Some experiments have shown improved performance following interruptions. ${ }^{15}$ Similarly, in clinical settings some interruptions are judged as appropriate and necessary. In a study of paediatric nurses who experienced over 5000 interruptions, $11 \%$ were assessed as having a positive outcome. $^{16}$ 
While multiple studies have measured the rate of interruptions to clinicians in hospitals, evidence of a direct link between interruptions and errors in healthcare remains surprisingly sparse. ${ }^{17}$ More tellingly, few studies have sought to test the effectiveness of specific interventions to reduce interruptions and error. In their study, Prakash et al ${ }^{18}$ make a valuable contribution by demonstrating the effects of interruptions on errors during chemotherapy drug verification and administration and testing a range of interventions. Their results confirm the association between interruptions and process errors. For example, interruptions were associated with a significant increase in failure to verify the volume in a syringe. Their experiment also demonstrated that simple, non-invasive interventions, such as the use of a large timer for intravenous pushes which provides nurses with a clear visual cue and assists in reminding nurses where they are up to in intravenous administration, can be effective in reducing errors.

Testing such interventions in a simulated environment is an important step prior to undertaking trials in real clinical settings. Currently, we only have limited evidence of the effectiveness and sustainability of other interventions utilised around the world, such as 'Do not interrupt' tabbards worn by nurses during medication rounds. ${ }^{19}$ Given our still limited understanding of the role of interruptions in supporting or hindering clinical work, we should proceed with caution in imposing largely untested interventions on the workforce.

Another productive area for research is consideration of interruptions in the broader socio-technical context. A limitation of research in this field has been a focus on interruptions as an isolated event when, in fact, it is the flow and accumulation of interruptions that are likely to influence their impact on individuals in terms of demands on memory and attention and consequently their effects on efficiency and error production. ${ }^{20}$ Is there a tipping point at which interruptions start to degrade performance ${ }^{21}$ By studying patterns of interruptions over time using direct observational techniques, and measuring performance against explicit task outcomes, we are more likely to glean new insights into current and possibly new strategies which may be effective in supporting clinicians in interruptive clinical environments. However, the methodological and theoretical challenges are significant and warrant greater attention. ${ }^{17}$

Likewise, it is important to take into account the influence of the social context on individuals' reactions to interruptions and the strategies they employ to deal with competing demands. ${ }^{22}$ Despite the global publicity received by books like CrazyBusy, many of us consider ourselves exceptional, not only remaining confident in our own ability to multi-task, but considering it a sign we are highly efficient and technically savvy. Each interruption presents a choice between dealing with the interrupting task or query by temporarily putting the original task aside, or attempting to multi-task by combining the new request with the original task. These options consume different cognitive resources and yet we know little about the consequences of adopting these different strategies, or how different social contexts mediate the choices people make. Given the common resort to multi-tasking in response to an interruption, this is a particularly fruitful area for further investigation.

Sanbonmatsu et $a l^{23}$ studied 210 US undergraduates, the majority of whom rated their multi-tasking ability as above average, and found that their multitasking performance was inferior to their perceptions of their corresponding multi-tasking skills. In particular, those most likely to report using a mobile phone while driving were found to be the least proficient when performing a multi-tasking skills test. In another study, a perhaps startling $16 \%$ of 706 US college students reported taking multi-tasking to a new level; admitting that they had engaged in sexual activities while also driving a moving vehicle. ${ }^{24}$ This was despite recognising the substantial risk of a car accident, with men estimating the risk of an accident at $56 \%$ and women at $74 \%$. Somewhat reassuringly, however, most respondents indicated that there was a low likelihood that they would engage in sexual activities while driving in the future.

The effects of more mundane multi-tasking are slowly being revealed. Just $e t \mathrm{al}^{25}$ studied the impact on drivers of concentrating on someone speaking. While performing a simulated driving task, subjects were played recorded statements and asked to judge them as true or false, to ensure they were listening attentively and driving. Using MRI, activations in the brain were also tracked during the simulation. Listening resulted in a significant degradation in driving performance-measured by deviations from the road and staying within the lane-and was associated with reduced activation in key parts of the brain previously shown to underpin driving. The researchers hypothesised that listening draws resources away from the task of driving, reducing activity in the areas of the brain that support performance for this task.

Such results clearly beg the question: 'are there important parallels in healthcare environments?' For example, how often do clinicians perform routine tasks that impose equivalent cognitive demands, say, to driving a car, while simultaneously listening to colleagues asking them questions? In healthcare settings it is so common it seems unremarkable to see doctors documenting or viewing information on a computer screen while on the phone or nurses being asked questions during a medication round. A direct observational study of 57 nurses over $191 \mathrm{~h}$ showed that of all tasks, nurses were most likely to be interrupted and to multi-task during medication administration, 
with $25 \%$ of all medication tasks undertaken while multi-tasking. ${ }^{7}$ Such behaviour seems on the surface efficient and often unavoidable, but may also have implications for safety.

Moving the research agenda forward requires the application of methods which allow us to distinguish interruption and multi-tasking behaviours which are crucial to safe care delivery, such as interrupting to prevent an incorrect drug administration, from those that may be harmful to performance. Currently, we know very little about the extent or effects of multitasking on clinical work and greater attention to the methodological challenges of investigating multitasking behaviours is required. Early work has demonstrated considerable variation between clinicians in their use of multi-tasking as a strategy to deal with incoming interruptions, including differences in performance depending on the time of day and the day of the week. ${ }^{26}$

As healthcare systems must respond to constantly changing, time-critical demands, there will always be many balls in play at the same time. But we need to be able to identify which particular balls demand undivided attention and when and how to intervene to shield clinicians from the chaotic bounces and ricochets of the rest.

\section{Competing interests None.}

Provenance and peer review Not commissioned; internally peer reviewed.

\section{REFERENCES}

1 Hallowell E. CrazyBusy: Overstretched, Overbooked, and About to Snap! Strategies for Handling Your Fast-Paced Life. USA: Random House, 2006.

2 Greenberg C, Regenbogen S, Studdert D, et al. Patterns of communication breakdowns resulting in injury to surgical patients. J Am Coll Surg 2007;204:533-40.

3 Reader T, Flin R, Cuthbertson B. Communication skills and error in the intensive care unit. Curr Opin Crit Care 2007;13:732-6.

4 Chisholm C, Collison K, Nelson D, et al. Emergency department workplace interruptions: are emergency physicians 'interrupt-driven' and 'multitasking'? Acad Emerg Med 2000;7:1239-43.

5 Westbrook J, Coiera E, Dunsmuir WTM, et al. The impact of interruptions on clinical task completion. Qual Saf Health Care 2010;19:284-9.

6 Westbrook JI, Ampt A, Kearney L, et al. All in a day's work: an observational study to quantify how and with whom doctors on hospital wards spend their time. Med J Aust 2008;188:506-9.

7 Westbrook J, Duffield C, Li L, et al. How much time do nurses have for patients? A longitudinal study of hospital nurses' patterns of task time distribution and interactions with other health professionals. BMC Health Serv Res 2011;11:319.
8 Ulanimo V, O’Leary-Kelley C, Connolly P. Nurses' perceptions of causes of medication errors and barriers to reporting. J Nurs Care Qual 2007;22:28-33.

9 Ly T, Korb-Wells C, Sumpton D, et al. Nature and impact of interruptions to clinical workflow of ward-based junior medical officers. J Graduate Med Educ 2013.

10 Westbrook J, Woods A, Rob MI, et al. Association of interruptions with increased risk and severity of medication administration errors. Arch Intern Med 2010;170:683-90.

11 Cellier J, Eyrolle H. Interference between switched tasks. Ergonomics 1992;35:25-36.

12 Monk CA. The effect of frequent versus infrequent interruptions on primary task resumption. Human Factors and Ergonomics Society 48th Annual Meeting, 2004.

13 Bailey B, Konstan J. On the need for attention-aware systems: measuring effects of interruption on task performance, error rate and affective state. Comput Hum Behav 2006;22:685-708.

14 Mark G, Gudith D, Klocke U. The cost of interrupted work: more speed and stress. In Computer-Human Interactions (CHI). Florence, Italy: ACM, 2008.

15 Zijlstra FRH, Roe RA. Temporal factors in mental work: Effects of interrupted activities. J Occup Organ Psychol 1999;72:163-85.

16 McGillis Hall L, Pedersen C, Hubley P, et al. Interruptions and pediatric patient safety. J Pediatr Nurs 2010;25:165-75.

17 Coiera E. The science of interruption. BMJ Qual Saf 2012;21:357-60.

18 Prakesh V, Koczmara C, Savage P, et al. Mitigating errors caused by interruptions during medication verification and administration: interventions in a simulated ambulatory chemotherapy setting. BMJ Qual Saf 2014;23:884-92.

19 Raban M, Westbrook J. Are interventions to reduce interruptions and errors during medication administration effective? A systematic review. BMJ Qual Saf 2014;23: 414-21.

20 Baethge A, Rigotti T, Roe R. Just more of the same, or different? An integrative theoretical framework for the study of cumulative interruptions at work. Euro J Work Organ Psychol 2014. Published Online First 2 April 2014.

21 Westbrook J. Interruptions to clinical work: How frequent is too frequent? J Graduate Med Educ 2013;5:337-9.

22 Harr R, Kaptelinin V. Unpacking the social dimension of external interruptions. In Proceedings of the International Association for Computing Machinery (ACM) Conference on Supporting Group Work, 2007.

23 Sanbonmatsu D, Strayer D, Medeiros-Ward N, et al. Who multi-tasks and why? Multi-tasking ability, perceived multi-tasking ability, impulsivity, and sensation seeking. PLoS One 2013;8:e54402.

24 Struckman-Johnson C, Gaster S, Struckman-Johnson D. A preliminary study of sexual activity as a distraction for young drivers. Accid Anal Prev 2014;71:120-8.

25 Just MA, Keller TA, Cynkar J. A decrease in brain activation associated with driving when listening to someone speak. Brain Res 2008;1205:70-80.

26 Walter SR, Li L, Dunsmuir WTM, et al. Managing competing demands through task-switching and multitasking: a multi-setting observational study of 200 clinicians over 1000 hours. BMJ Qual Saf 2014;23:231-41. 